\title{
NOTICES SUR LES AUTEURS ET ADRESSES
}

Dominique BouRel, né en 1952, est directeur de recherche au CNRS. Il a été rédacteur en chef de la Revue de synthèse de 1992 à 1995 et dirige actuellement le Centre de recherche français de Jérusalem. Ses travaux portent sur l'histoire culturelle de l'Europe moderne.

Adresse: Centre de recherche français de Jérusalem, 5 rue Shimshon, BP 547, 91004 Jérusalem (Israël).

Enrico Castelli Gattinara, né en 1959, maître de conférences associé à l'EHESS, Paris, est spécialiste de la philosophie et de l'épistémologie française du $\mathrm{xx}^{\mathrm{e}}$ siècle. Il est, en outre, rédacteur en chef de la revue La Ginestra. Quaderni di cultura psicanalitica.

Adresse : Via Antonio Allegri de Corregio, 11, I-00196 Rome.

Bertrand MüLLER, né en 1955, chargé de recherches à l'université de Lausanne, s'est spécialisé dans le domaine de l'histoire de l'histoire et de l'histoire des sciences sociales. Rédacteur de la Revue suisse d'histoire et co-fondateur des Cahiers Marc Bloch, il a publié récemment le premier volume de la Correspondance Marc Bloch-Lucien Febvre (Paris, Fayard, 1994).

Adresse : Université de Lausanne, BF SH2-ISP-bureau 5119, CH-1015 Lausanne.

Christophe Prochasson est maître de conférences à l'École des hautes études en sciences sociales. Il collabore aux revues Le Mouvement social et Mil neuf cent. Revue d'histoire intellectuelle. Il a publié, entre autres, Les Années électriques, 1880-1910 (Paris, La Découverte, 1991), Les Intellectuels, le Socialisme et la Guerre (Paris, Seuil, 1993), Au nom de la Patrie. Les intellectuels et la Première Guerre mondiale, 1910-1919 (Paris, La Découverte, 1996).

Adresse: Centre de recherches historiques, École des hautes études en sciences sociales, 54 bd Raspail, 75006 Paris.

Laurent MuCCHIELLI, né en 1968, est chargé de mission à la Fondation nationale des sciences politiques. Il travaille sur l'histoire des sciences humaines aux $\mathrm{xIX}^{\mathrm{e}}$ et $\mathrm{XX}^{\mathrm{e}}$ siècles. Adresse : 26, rue Au Maire, 75003 Paris.

Revue de synthèse : $4^{\mathrm{e}} \mathrm{S}$. N ${ }^{\text {os }}$ 3-4, juil.-déc. 1996, p. 615-617. 
Charel B. KRoL, né en 1960 à La Haye (Pays-Bas), a soutenu sa thèse de doctorat en droit, en 1994, à l'université de Maastricht. Il est actuellement professeur de European Studies à l'université d'Odessa.

Adresse : Odessa State University, Ul. Petra Velikogo 2, 270100 Odessa (Ukraine).

Michel BLAY, directeur de recherche au CNRS et rédacteur en chef de la Revue d'histoire des sciences, est né à Paris en 1948. Ses recherches portent principalement sur l'histoire de l'optique et de la mécanique aux XVII ${ }^{e}$ et XvIII ${ }^{\mathrm{e}}$ siècles. Parmi ses dernières publications, on peut signaler, La Naissance de la mécanique analytique (Paris, Presses universitaires de France, 1992), Les Raisons de l'infini; du monde clos à l'univers mathématique (Paris, Gallimard-Essais, 1993), Les Figures de l'arc-en-ciel (Paris, Carré, 1995).

Adresse: Centre international de synthèse, 12 rue Colbert, 75002 Paris.

Pietro REDONDI, né en 1950, est maître de conférences en histoire des sciences à l'université de Bologne et travaille sur la physique classique : Galilée, optique physique, technologie.

Adresse : Università degli studi di Bologna, Dipartimento di filosofia, via Zamboni, 38, I-40126 Bologne.

Jacques Revel, né en 1942, est directeur d'études à l'École des hautes études en sciences sociales. Il travaille actuellement sur l'histoire des relations entre la discipline historique et les sciences sociales depuis la fin du $\mathrm{xIx}^{\mathrm{e}}$ siècle.

Adresse : École des hautes études en sciences sociales, 54 bd Raspail, 75006 Paris.

Martin Fugler, né en 1961, professeur d'histoire en lycée professionnel à Strasbourg et formateur à l'IUFM d'Alsace, est membre de différents groupes de travail sur la didactique de l'histoire.

Adresse : 13 rue de Bruxelles, 67000 Strasbourg.

Peter SCHÖTtLER, né en 1950, est docteur en histoire. Chargé de recherche au CNRS depuis 1989, il est membre du centre Marc-Bloch de Berlin (1995-1996) et visiting fellow (1996-1997) à l'université de Princeton. Ses domaines de recherche sont l'histoire culturelle franco-allemande, l'histoire du nazisme et l'histoire de l'histoire.

Adresse : Centre Marc-Bloch, Schiffbauerdamm 19, D-10117 Berlin.

Marina NERI, né en 1966, prépare une thèse à l'École des hautes études en sciences sociales sous la direction de Jacques Revel. Elle étudie l'historiographie française de l'entre-deux-guerres et tout particulièrement les modalités de réception et d'application de certaine notions psychologiques (sensibilité, mentalité, etc.) dans le domaine historique, spécifiquement dans les travaux des fondateurs des Annales, Lucien Febvre et Marc Bloch. Actuellement, elle prépare aussi l'édition française et italienne de la correspondance entre Lévy-Bruhl et Moisei Ostrogorski, ainsi qu'une étude sur la biographie intellectuelle du politologue russe et les rapports entre les communautés intellectuelles juives russe et française.

Adresse: Regensburger Str. 32, D-10777 Berlin.

Bernadette Bensaude-Vincent, née en 1949, est professeur à l'université Paris X. Auteur de Paul Langevin. Science et vigilance (Paris, Belin, 1987), elle a publié également plusieurs articles et ouvrages sur l'histoire de la chimie.

Adresse: Université Paris X-Nanterre, 200 av. de la République, 92001 Nanterre Cedex. 
Margherita Platania, née à Naples en 1948, est maître de conférences à la faculté des lettres de l'université de Salerne. Directeur d'études associé en histoire sociale (19931995 ) et en histoire contemporaine (1995-1996) de la même université, elle consacre essentiellement ses recherches à l'historiographie française et à l'histoire sociale de l'Italie.

Adresse: Viale dei Tigli 2, I-80131 Naples.

Jacqueline Pluet-Despatin, née en 1935, docteur en histoire, ingénieur de recherche à la Maison des sciences de l'homme, chargée de mission à l'Institut Mémoires de l'édition contemporaine, est spécialiste de l'histoire des revues et de l'édition.

Adresse: Institut Mémoires de l'édition contemporaine, 25 rue de Lille, 75007 Paris.

Giuliana GEMELLI, née en 1952, est professeur d'histoire contemporaine française à l'université de Bologne. Elle est aussi président du Comité scientifique de l'Institut de recherches européennes de l'université de Bologne. Son champ principal de recherche est l'historiographie et l'histoire des institutions scientifiques dans les sciences sociales, l'économie et le management en France, en Italie et aux États-Unis. Elle publie actuellement une histoire sociale des sciences sociales dans la France contemporaine chez l'éditeur Il Mulino.

Adresse : Dipartimento di discipline storiche, Piazza San Giovanni 2, I-40124 Bologne.

Yannick MaIGNiEn, né en 1950, est responsable de la Mission pour la politique scientifique de numérisation de la Bibliothèque nationale de France où il mène une réflexion sur le rôle des documents structurés dans les collections numérisées.

Adresse: Bibliothèque nationale de France, 11 quai François-Mauriac, 75706 Paris Cedex 13.

Éric DusserT, né en 1967, est responsable d'acquisition dans le même service pour les disciplines économique, juridique et politique. Il poursuit des recherches bibliographiques relatives à l'histoire de l'édition française au $\mathrm{xx}^{\mathrm{e}}$ siècle.

Adresse: Bibliothèque nationale de France, 11 quai François-Mauriac, 75706 Paris Cedex 13. 